\title{
Cryopreservation of single-sperm: where are we today?
}

Shasha Liu' ${ }^{1,2}$ and Fuping $\mathrm{Li}^{1,2^{*}}$ (D)

\begin{abstract}
Background: Patients with severe oligospermia and nonobstructive azoospermia have very limited numbers of viable sperm in their epididymal and testicular samples. Thus, cryopreservation of their sperm is performed to avoid repeated sperm retrievals and to preserve their sperm from any side effects of any treatment regimens.

Main body: The development of intracytoplasmic sperm injection technology has extended the therapeutic capacity of assisted reproductive technology for men with azoospermia via the surgical or percutaneous isolation of sperm from the testis/epididymis. The conventional cryopreservation techniques are inadequate for preserving individually selected sperm. The technique for freezing single sperm was first developed in 1997 and has been explored from the perspective of frozen carriers, freezing programs, and cryoprotectant formulations. Among these methods, advances in frozen carriers have directly improved single-sperm freezing technology. In this review, we evaluate the different technologies for the cryopreservation of single sperm by discussing the advantages and disadvantages of different freezing methods, their clinical applications, and the outcomes for a range of frozen carriers.

Conclusion: Our review article describes the latest and current technologies implemented for the cryopreservation of single sperm that could potentially benefit patients with severe oligospermia and who rarely have any sperm in their ejaculate. This review provides a platform to understand the process and pitfalls of single-sperm cryopreservation to ensure further improvements in the cryopreservation technology in future studies.
\end{abstract}

Keywords: Cryopreservation, Single sperm, Frozen carrier, Freezing method, Clinical application

\section{Background}

In recent years, increasing environmental pollution owing to continuous industrialization and psychological stresses $[1,2]$ have resulted in an increase in infertility issues worldwide. Infertility in men now accounts for approximately $50 \%$ of the fertility-related problems [3]. Infertility is frequently observed in patients with cancer and in men with severe oligozoospermia or azoospermia owing to spermatogenic dysfunction. The primary challenge in clinical practice is ensuring that the patient is

\footnotetext{
* Correspondence: Ifpsnake@scu.edu.cn

${ }^{1}$ Human Sperm Bank, West China Second University Hospital, Sichuan University, Chengdu 610041, China

${ }^{2}$ Key Laboratory of Birth Defects and Related Disease of Women and Children (Sichuan University), Ministry of Education, Chengdu 610041, China
}

restored to good health after receiving the relevant treatments. However, specific treatment regimens (chemotherapy, radiotherapy, surgery) lead to adverse side effects such as impaired spermatogenesis, testosterone deficiency, or sexual dysfunction, thereby affecting the reproductive and psychological well-being of the patient $[4,5]$. Most patients are concerned about the fertilityrelated side effects, regardless of the treatments received. Therefore, preserving fertility before treatment is critical to ensure the quality of life. In addition, etiological factors involved in male infertility factors also include congenital factors (anorchia, cryptorchidism, congenital absence of vas deferens, genetic abnormalities), acquired factors (testicular torsion, testis trauma, surgery etc.) and idiopathic forms. The etiology is still unknown in 
about half of the cases and it is termed "idiopathic infertility" [6]. The complete diagnostic workup is important for patients. In general, cryopreservation of sperm is a way to avoid risks.

Sperm banking was initially used for the cryopreservation of sperm [7] and was first developed to facilitate pregnancy in the 1950s [8]. Cryopreservation technologies are integral for managing male-factor infertility. Sperm donors are properly screened and quarantined before semen cryopreservation to eliminate the risk of transmission of HIV, hepatitis B and C, syphilis, and bacterial infections to the recipient. Advancements in intracytoplasmic sperm injection (ICSI) technology provide the opportunity to overcome fertility issues in patients with azoospermia (when the semen is devoid of sperm) and severe oligozoospermia. The ability to cryopreserve small numbers of spermatozoa in males afflicted with nonobstructive azoospermia using testicular sperm extraction (TESE) avoids the requirement for repeated surgery and promotes the preservation of fertility [9-13]. Conventional sperm preservation techniques can result in sperm loss owing to sperm adherence to the carrier vessel, harsh centrifugation, and washing procedures [14]. Thus, the conventional technique is particularly problematic in cases in which sperm numbers are low [15].

In 1997, Cohen et al. were the first to describe a novel cryopreservation technique for individual sperms using an empty zona pellucida (ZP) [16], thereby providing a theoretical and technical foundation for subsequent techniques. They introduced the concept of singlesperm freezing. Since then, a multitude of cryopreservation techniques have been developed to improve sperm counts. In this article, we review the single-sperm cryopreservation methods practiced in the last 20 years (Table 1), including the types of frozen carriers (Table 2), spermatozoa from different sources, prefreezing treatments, freezing procedures, resuscitation after freezing, and clinical applications. We performed an in-depth analysis of the advantages and disadvantages of these techniques to provide the theoretical basis for improving the single-sperm freezing technology in the future.

\section{Single-sperm cryopreservation within the empty ZP}

Repeated TESE is both costly and invasive, and it can frequently have adverse effects on the testis, including deterioration of spermatogenesis, inflammation, irreversible atrophy, and partial testicular devascularization [12]. Repetition of these procedures can be avoided through the cryopreservation of spermatozoa. Although studies $[44,45]$ have reported sperm survival and the births of live offspring following the cryopreservation of epididymal and testicular sperm, conventional sperm freezing techniques result in low viability [46]. Therefore, it is crucial to develop new sperm freezing methods. The loss of spermatozoa through conventional addition and removal of cryoprotectants in relatively large volumes of media can be circumvented by the insertion of the spermatozoon into an enclosed porous capsule that can be correctly visualized and handled microscopically prior to and after cryopreservation. The chosen vehicle for such a purpose is the ZP that can be used following the removal of its cellular material. The cytoplasmic components in the oocyte $\mathrm{ZP}$ were removed, and individual sperm was injected into the empty ZP using an ICSI needle and loaded onto a frozen straw for cryopreservation (Fig. 1a). After thawing, the recovery and fertilization rates were $73 \%$ and $50 \%$, respectively. Spermatozoa recovered from human zonae fertilized the same proportion of oocytes as fresh fertile control spermatozoa. The fertilization rate in human zonae was marginally lower $(P<0.05)$ than that for spermatozoa frozen in animal zonae [16]. Over the next 6 years, frozen vectors from the empty ZP of human, mice, hamsters, and golden hamsters were used [16-23, 47, 48]. There was no statistical difference in freezing and thawing results of human and mouse ZP as carriers [20,47]. In 1998, Walmsley et al. [17] reported the first cases of live births after ICSI procedures by using empty ZP frozen testicular sperm.

The empty ZP is larger than the sperm, and it forms a transparent matrix that is easy to observe and manipulate through microscopy. However, inevitable shortcomings limited their development and ZP was replaced later with non-biological carriers. The empty ZP of animals acts as a biological carrier, but it has limited availability and bioethical issues. New FDA and European Tissue Directive regulations regarding the exposure of human gametes and embryos to animal products make the use of rodent zonae less feasible. Furthermore, human zonae availability is also very restricted. To use an empty $\mathrm{ZP}$ as a frozen carrier, a hole is punched in the ZP using a laser. However, drilling small holes can increase the risk of leaving some host DNA fragments behind. The transfer of foreign DNA using sperm as vectors during ICSI has been reported previously $[49,50]$. On the other hand, drilling larger holes can lead to the risk of sperm loss. Furthermore, attempts to seal the hole using oil droplets has limited effectiveness and interferes with sperm recovery post-thaw $[18,23]$. Sperm and ZP3 binding on the human ZP also induces acrosome reactions, thereby frequently affecting sperm quality [16].

\section{Single-sperm cryopreservation within the Volvox globator sphere}

The Volvox globator is an alternative in countries that prohibit the destructive use of oocytes, even after fertilization has failed. In 2004, Just et al. [24] used the 
Table 1 Single-sperm cryopreservation methods

\begin{tabular}{|c|c|c|c|c|c|c|}
\hline Carrier & Year & Sperm origin & $\begin{array}{l}\text { Recovery } \\
\text { rate }\end{array}$ & $\begin{array}{l}\text { Motility } \\
\text { rate }\end{array}$ & $\begin{array}{l}\text { Survival } \\
\text { rate }\end{array}$ & $\begin{array}{l}\text { Fertilization } \\
\text { rate }\end{array}$ \\
\hline Human, mouse and hamster zonae [16] & 1997 & Ejaculate & $73 \%$ & $82 \%$ & NA & $50 \%$ \\
\hline Human and hamster zonae [17] & 1998 & Ejaculate, testicular & $\geq 75 \%$ & $67-100 \%$ & NA & $65 \%$ \\
\hline Human zona [18] & 1999 & Ejaculate & $92 \%$ & NA & $84 \%$ & NA \\
\hline Human zonae [19] & 2000 & Testicular & $88 \%$ & $27 \%$ & NA & $23 \%$ \\
\hline Human and mouse zonae [20] & 2000 & Ejaculate, epidydimal, testicular & $82 \%$ & $83 \%$ & NA & NA \\
\hline Mouse zonae [21] & 2000 & Testicular & $100 \%$ & $58 \%$ & $77 \%$ & NA \\
\hline Human zona pellucida [22] & 2001 & Epidydimal & NA & NA & NA & $57 \%$ \\
\hline Human zona [23] & 2003 & Ejaculate & $59 \%$ & $73 \%$ & NA & NA \\
\hline Volvox globator algae [24] & 2004 & Ejaculate & $100 \%$ & $\geq 63 \%$ & NA & NA \\
\hline Alginic acid drops [25] & 2006 & Ejaculate & NA & $20-30 \%$ & $40-50 \%$ & NA \\
\hline Agarose gel microspheres [26] & 2007 & Ejaculate & $98 \%$ & $78 \%$ & $81 \%$ & NA \\
\hline Hollow-core agarose capsules [27] & 2015 & Ejaculate & $94 \%$ & $84 \%$ & $95 \%$ & NA \\
\hline Hollow hyaluronan-phenolic hydroxyl microcapsules [28] & 2016 & Ejaculate & $95 \%$ & $14 \%$ & NA & NA \\
\hline Cryoloops [29] & 2003 & Ejaculate & NA & $45 \%$ & NA & NA \\
\hline Cryoloops [30] & 2004 & Ejaculate & $68 \%$ & $73 \%$ & NA & $67 \%$ \\
\hline Cryoloop [31] & 2004 & Epidydimal, testicular & $72 \%$ & NA & NA & $58 \%$ \\
\hline Droplets on plastic dish [32] & 2000 & NA & $90-100 \%$ & NA & NA & NA \\
\hline 0.5ul microdrop in a plastic dish [33] & 2003 & Ejaculate & $100 \%$ & $<50 \%$ & NA & NA \\
\hline 5ul microdrop in a plastic dish [34] & 2008 & Testicular & $100 \%$ & $2 \%$ & NA & $18 \%$ \\
\hline Cell Sleeper [35] & 2012 & Testicular & $83 \%$ & NA & NA & $83 \%$ \\
\hline Cell Sleeper [36] & 2012 & Ejaculate & $100 \%$ & $28 \%$ & $58 \%$ & NA \\
\hline Cell Sleeper [37] & 2016 & Testicular & $94 \%$ & $56 \%$ & NA & $66 \%$ \\
\hline Cryotop [35] & 2012 & Ejaculate, testicular & $96 \%$ & NA & NA & $64 \%$ \\
\hline Cryotop [36] & 2012 & Ejaculate & $100 \%$ & $44 \%$ & $78 \%$ & NA \\
\hline Cryotop [38] & 2011 & Ejaculate, testicular & $93 \%$ & $43 \%$ & NA & NA \\
\hline Cryoleaf [39] & 2011 & Ejaculate, epididymal & $99 \%$ & $66 \%$ & NA & $61 \%$ \\
\hline Cryopiece [40] & 2017 & Ejaculate, testicular & $83 \%$ & $48 \%$ & NA & $73 \%$ \\
\hline Closed slice [41] & 2015 & Ejaculate & $94 \%$ & $41 \%$ & $70 \%$ & NA \\
\hline Closed slice [42] & 2017 & Testicular & $92 \%$ & $17 \%$ & $66 \%$ & $56 \%$ \\
\hline The novel sperm vitrification device [43] & 2018 & Ejaculate & $96 \%$ & $33 \%$ & NA & $59 \%$ \\
\hline
\end{tabular}

Volvox globator as a single-sperm cryopreservation vehicle (Fig. 1b). Spherical colonies (diameter 0.5-1.0 mm) contain 1500-20,000 peripheral cells. Considering its size and green color, the Volvox globator is easy to identify and operate. Eight sperm were frozen in each algae ball, and three Volvox spheres were placed in a frozen straw at $4{ }^{\circ} \mathrm{C}$ for $10 \mathrm{~min}$. The straws were frozen on liquid nitrogen $\left(\mathrm{LN}_{2}\right)$ vapor for $10 \mathrm{~min}$ before submerging in $\mathrm{LN}_{2}$. The spermatozoa recovery rate was $100 \%$, and motility rate was at least $60 \%$ after thawing. The use of the spherical algae Volvox globator offered a promising approach to the cryopreservation of functional motile sperm. A drawback of the Volvox sphere was the transfer of genetic material from the algae to the egg cells, which requires further investigation. Moreover, the new FDA and European Tissue Directive regulations consider the use of algae (non-human tissue) to store human sperm unacceptable in a clinical setting.

\section{Single-sperm cryopreservation within non-biological carriers such as empty ZP}

Sperm injection into an empty ZP is easier for technicians because the procedure is similar to that of ICSI [15]. However, concerns remain regarding the use of ZP, including bacterial and virus contaminants from animals. To overcome these challenges, hollow capsules similar to empty ZP have been fabricated to freeze small numbers of sperms. 
Table 2 Summary of the different type carriers for single-sperm cryopreservation

\begin{tabular}{ll}
\hline Biological & Non-biological \\
\hline Zona pellucida of different species & Polymerized alginic acid capsules \\
Volvoxator algae & Hollow-core agarose capsules \\
& Hollow hyaluronan-phenolic hydroxyl microcapsules \\
Cryoloops & Culture dish \\
Cell Sleeper & Cryotop \\
Cryoleaf \\
Cryopiece \\
Closed slice \\
The novel sperm vitrification device
\end{tabular}

\section{Polymerized alginic acid capsules}

The empty capsule of a non-biological carrier is similar to empty ZP obtained from an animal. In 2006, Herrler et al. [25] developed a method of freezing small amounts of spermatozoa in polymerized alginic acid drops. Alginic acid is a chemically inert mixture of two sugars, namely, $\beta$-D-mannuronic and $\alpha$-L-guluronic acid. Alginate capsules containing sperm were placed in cryoprotective agent (CPA) and frozen in straws using a programmable freezer. On thawing, alginate beads were dissolved in a sodium citrate solution. Alginate is a nontoxic polysaccharide, and polymerized alginic acid beads have been successfully used for the cryopreservation of hepatocytes [51] and stem cells [52]. The advantages of using alginate as a single-sperm freezing carrier are its gel liquid properties and its chemical inertness. Cryopreservation of human sperm by this protocol resulted in a decreased motility of about $20 \%$ compared with standard protocols. This was most likely caused by the alginic acid covering the surface of the sperm [25]. Owing to the complexity of the entire operation and other shortcomings, the developments in this technology are slow, thereby limiting its clinical application.

\section{Hollow-core agarose capsules}

In the last decade, the use of empty capsules for singlesperm freezing has developed slowly. In 2007, Isaev et al. [26] used empty agarose microspheres (100- $\mu \mathrm{m}$ diameter) as a non-biological analog to ZP. One to ten spermatozoa were added into each agarose microsphere and placed in CPA solution for $5 \mathrm{~min}$. One to five agarose microspheres were put into a $250 \mu \mathrm{L}$ plastic straw. The straws were exposed to $\mathrm{LN}_{2}$ vapor for $10 \mathrm{~min}$ and then plunged into the $\mathrm{LN}_{2}$. After thawing, the sperm recovery rate was $98 \%$, and $78 \%$ of recovered spermatozoa were motile. The supravital rate was $81 \%$. In 2015, Araki et al. [27] designed hollow-core agarose capsules of size comparable with the mammalian oocytes (Fig. 2a), which were effective cryopreservation methods for individual sperm. Agarose gels had a mesh structure [53] with the advantage of being able to adjust the volume of solution on the sheet for ease-of-operation. Each agarose gel was a

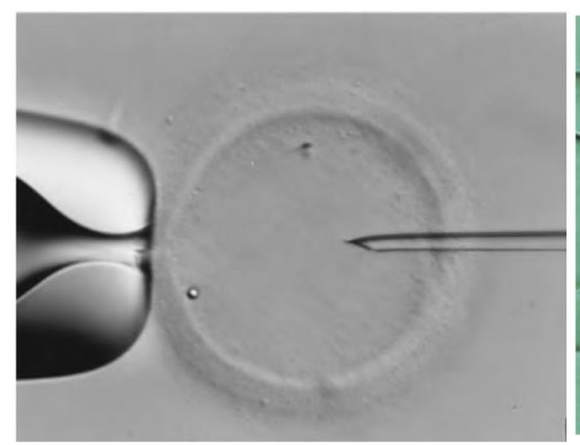

b

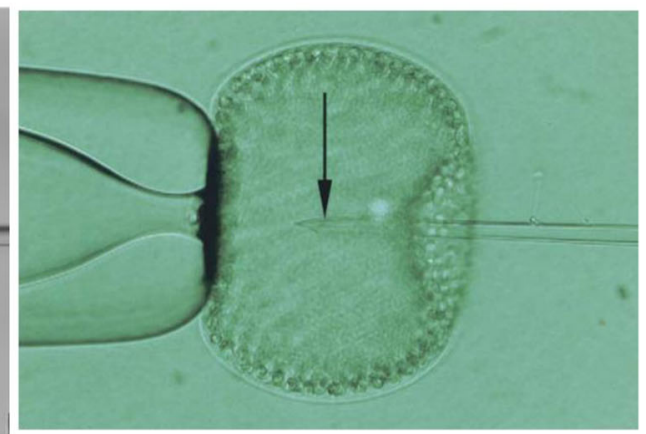

Fig. 1 (a) (Adapted from Cohen et al. [16]) Empty pre-fertilization human zona. The needle was inserted into the zona. (b) (Adapted from Just et al. [24]) Volvox globator sphere, fixed to the holding pipette 
a

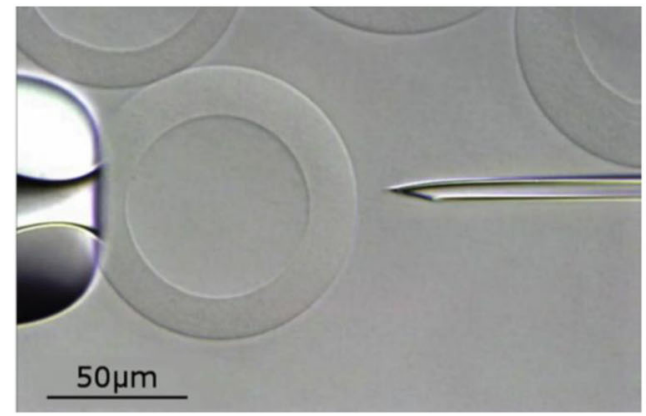

b

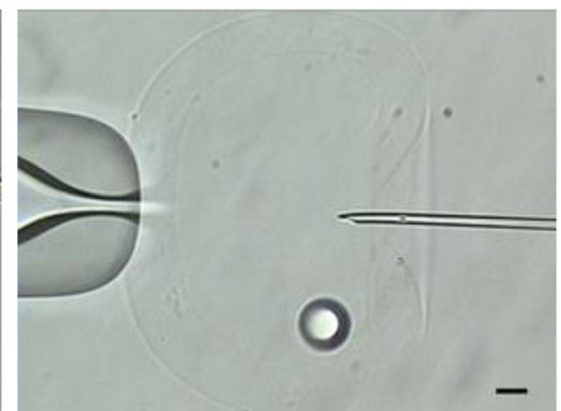

Fig. 2 (a) (Adapted from Araki et al. [27]) Hollow-core agarose capsule (outer diameters, 80-120 4 m; inner diameters, 60-100 4 m) held by a holding pipette before injection. (b) (Adapted from Tomita et al. [28]) The needle was inserted into the hollow hyaluronan-phenolic hydroxyl microcapsules and one spermatozoon was slowly released. The average diameter of capsules was $241.6 \pm 30.4 \mu \mathrm{m}$ and thickness of the HA membrane was $29.6 \pm 7.2 \mu \mathrm{m}(N=38)$. Scale bars $=20 \mu \mathrm{m}$

loaded with one sperm and placed onto the mesh or polycarbonate sheets containing 0.25 to $0.5 \mu \mathrm{L}$ cryoprotectant solution. They were frozen in $\mathrm{LN}_{2}$ vapor for 10 $30 \mathrm{~s}$ and then immersed into $\mathrm{LN}_{2}$. Sperm resuscitation was performed in mineral oil at $37^{\circ} \mathrm{C}$. The recovery and survival rates of the sperm exceeded $90 \%$, with activity rate of $\geq 80 \%$. Spermatozoa were identified over a short time frame $(10 \mathrm{~s})$ after thawing, and they remained in the capsule. Agarose gel materials exhibit low toxicity, and therefore permit the culture of mammalian cells [54]. The hollow-core agarose capsules are relatively new and require further trials and investigations. More sperm function testing data will be needed to assess a potential carrier for clinical application.

\section{Hollow hyaluronan-phenolic hydroxyl microcapsules}

In 2016, Tomita et al. [28] showed that hollow hyaluronan -phenolic hydroxyl (HA-Ph) microcapsules could be used for the cryopreservation of small numbers of sperms with no adverse effects on sperm quality (Fig. 2b). Three sperm were injected into a hollow HA microcapsule using a micromanipulator. Capsules were placed into Cryotop devices containing $1 \mu \mathrm{L}$ of cryoprotectant. Cryotop devices were placed at $4.5 \mathrm{~cm}$ above the $\mathrm{LN}_{2}$ vapor for $2 \mathrm{~min}$ and plunged into $\mathrm{LN}_{2}$. After thawing, all frozen microcapsules were recovered, and most of the sperm were recovered in hyaluronidase-decomposing HA-Ph gels after only 2.5 min. The sperm recovery rate was $95 \%$, which was higher than that of the empty ZP carrier. The proportion of active sperm was $14 \%$ after the resuscitation. The study showed that hollow HA-Ph microcapsules with diameter differences of several tens of micrometers do not influence sperm motility after freeze-thawing [28]. Hyaluronidase does not influence sperm motility. The recovery rates of motile sperm were relatively low owing to the freezing of the aggregated gelatin, thereby causing damage to the sperm plasma membranes [15]. Recovering spermatozoa through degradation of the capsules is favorable because it prevents physical damage to the nuclear structure.

HA-Hp microcapsules represent non-biological carriers, no ethical problems exist. However, knowledge of the clinical outcomes of this procedure is lacking, and further studies in this area are required.

\section{Single-sperm cryopreservation within non-biological carriers (vitrification devices)}

In the past 20 years, the rapid development of the microdrop single-sperm freezing methods has led to the design and invention of various carriers. The use of suitable cryoprotectants (Table 3) can achieve better freezing effect. It is beneficial to clinical use.

\section{Cryoloops}

Cryopreservation of individual spermatozoa is a challenging task. Owing to the shortcomings of the empty ZP method, an effective non-labor intensive methodology was developed for the cryopreservation of individual spermatozoa. Using an open cryoloop enclosed in a vial could be an alternative option (Fig. 3a). Cryoloops have been employed with success for oocyte and embryo vitrification procedures [56-59], and Schuster et al. [29] used cryoloops for the cryopreservation of small aliquots of spermatozoa. In this method, spermatozoa were submerged in experimental test yolk buffer supplemented with $12 \% \mathrm{v} / \mathrm{v}$ glycerol, followed by freezing in $\mathrm{LN}_{2}$ vapor for $5 \mathrm{~min}$ and snap freezing in $\mathrm{LN}_{2}$. Cryoloop-mediated "ultra-rapid freezing" of the oligo sperm is both simple and fast, and in this study, it resulted in $45 \%$ motility rate [29]. Moreover, successful vitrification of human spermatozoa has been achieved on cryoloops in the absence of cryoprotectants $[14,55]$ and this technique can be easily implemented. The post-thaw viability has been reported to be $52 \%$, and sperm function was retained [14]. The DNA integrity and motility of the vitrified 
Table 3 Summary of non-biological carriers (vitrification devices) and cryoprotectants

\begin{tabular}{|c|c|}
\hline $\begin{array}{l}\text { Non-biological carriers } \\
\text { (Vitrification devices) }\end{array}$ & Cryoprotectants \\
\hline Cryoloops & $\begin{array}{l}\text { Test yolk buffer (TYB) with 12\% v/v glycerol (Irvine Scientific, USA) [29]. } \\
\text { With or without glycerol. Test-egg yolk-glycerol (TEYG) freezing medium (Scandinavian IVF Science, Gothenburg, } \\
\text { Sweden) (glycerol concentration 12\%); Standard medium (no cryoprotectant) [55]. } \\
\text { A 50:50 mixture of HTF-HEPES with 6\% plasmanate and the TYB-glycerol (Irvine Scientific, USA) cryoprotectant } \\
\text { [30]. } \\
\text { A 50:50 mixture of TYB-glycerol (Irvine Scientific, USA) and m-HTF medium supplemented with 6\% Plasmanate } \\
\text { [31]. }\end{array}$ \\
\hline Culture dish & $\begin{array}{l}\text { A 50:50 mixture of IVF-20 medium (JCD, France) and sperm freezing medium (Medicult, Denmark) [33]. } \\
\text { Sperm freezing medium (Medicult, Denmark) [34]. }\end{array}$ \\
\hline Cell Sleeper & $\begin{array}{l}\text { A mixture of } 0.7 \mathrm{~mL} \text { SpermFreeze (FertiPro, Belguim) and } 1.0 \mathrm{~mL} \text { HFF99 (Fuso Pharmaceutical Industries, Japan) } \\
\text { containing 20\% serum substitute supplement (SSS) (Irvine Scientific, USA) [35]. } \\
\text { SpermFreeze (FertiPro, Belgium) containing 20\% SSS (Irvine Scientific, USA) [36]. } \\
\text { A 50:50 mixture of SpermFreeze solution (Vitrolife, Goteborg, Sweden) and human serum albumin (HSA) } \\
\text { supplemented (10 } \mathrm{mg} \mathrm{ml}^{-1} \text {, by manufacturer) Sydney IVF Gamete Buffer (Cook Medical) [37]. }\end{array}$ \\
\hline Cryotop & $\begin{array}{l}\text { A mixture of } 0.7 \mathrm{~mL} \text { SpermFreeze (FertiPro, Belguim) and } 1.0 \mathrm{~mL} \text { HFF99 (Fuso Pharmaceutical Industries, Japan) } \\
\text { containing } 20 \% \text { SSS (Irvine Scientific, USA) [35]. } \\
\text { With or without glycerol. Two different cryoprotectants, sucrose (Sigma, USA) and SpermFreeze (FertiPro, Belguim) } \\
\text { were tested. SpermFreeze includes both glycerol and sucrose. The sucrose-based freezing medium was 0.1 M } \\
\text { sucrose in HFF99 (Fuso Pharmaceutical Industries, Japan) containing } 20 \% \text { SSS (Irvine Scientific, USA). SpermFreeze- } \\
\text { based freezing medium was a mixture of SpermFreeze (0.7 mL) and HFF99 (1.0 mL) containing 20\% SSS [38]. }\end{array}$ \\
\hline Cryoleaf & $12 \%(\mathrm{v} / \mathrm{v})$ glycerol and $20 \%(\mathrm{v} / \mathrm{v})$ egg yolk in $0.1 \mathrm{M}$ citrate buffer $(\mathrm{PH}=7.2)$ [39]. \\
\hline Cryopiece & Freezing medium used [40]. \\
\hline Closed slice & $\begin{array}{l}\text { With or without cryoprotectant. A 50:50 mixture of commercial sperm cryoprotectant (Quinn's Advantage SAGE, } \\
\text { USA) and HEPES buffer with 10\% HSA. The without cryoprotectant group used HEPES buffer with 10\% HSA [41]. } \\
\text { HEPES buffer with 10\% HSA [42]. }\end{array}$ \\
\hline $\begin{array}{l}\text { The novel sperm vitrification } \\
\text { device }\end{array}$ & $\begin{array}{l}\text { A 50:50 mixture of Quinn's Advantage Sperm Freezing Medium (SAGE, USA) and Quinn's Sperm Washing Medium } \\
\text { [43]. }\end{array}$ \\
\hline
\end{tabular}

spermatozoa frozen on cryoloops were shown to be comparable with that observed for conventionally frozen spermatozoa [55]. To our knowledge, no pregnancies have been reported using vitrified/warmed sperm.

In 2004, Desai et al. [30, 31] proposed a novel cryoloop method as an alternative to hamster zona for freezing individual sperm. In this study, the authors developed a nylon cryoloop to cryopreserve 5-10 ejaculated spermatozoa, with a total of 77 spermatozoa frozen in 10 cryoloops. After thawing, the recovery and motility rates were $68 \%$ and $73 \%$, respectively. No difference in postthaw motility was observed after cryopreservation on loops compared with conventional vials. These individually cryopreserved sperm were indeed capable of inducing sperm head decondensation and pronuclear formation when injected into human oocytes [30]. Each nylon cryoloop was also developed to cryopreserve 2-8 epididymal or testicular spermatozoa, and they achieved $72 \%$ recovery. Sperm motility involved minimal head motion and the cryopreserved epididymal and testicular spermatozoa (including non-motile sperms) could fertilize oocytes by using conventional ICSI methods [31].

Cryoloops can be commercially purchased and they require no additional preparation. Because no animal products are required for cryoloops and the materials are non-biological, bioethical problems are avoided, making it more favorable than the hamster ZP. However, the ventilation ports of the cryovials do act as open carriers, thereby posing a risk of $\mathrm{LN}_{2}$ leakage and crosscontamination. Frozen droplets were carried on the surface film, thereby making the storage system unstable. So far, this method has rarely been used.

\section{Culture dish}

Another simple method of freezing involves the replacement of the ZP by a small drop of freezing medium and its placement in a culture dish to be frozen (Fig. 3b) [32-34]. In 2000, Quintans et al. [32] cryopreserved 4-6 spermatozoa in a microdroplet with oil overlay in a plastic tissue culture dish. The dishes were sealed and stored in $\mathrm{LN}_{2}$, and the post-thaw recovery rate was $90-100 \%$ [32]. In 2003, Bouamama et al. [33] described singlesperm cryopreservation in culture dishes using microdroplet freezing. In this procedure, 1-100 spermatozoa were added to freezing media-microdroplet (volume: $0.5 \mu \mathrm{L}$ ) in paraffin oil, and the culture dish was sealed and stored in $\mathrm{LN}_{2}$. After thawing, complete (100\%) recovery rate was observed and sperm movement rate was $50 \%$. Sperm function was not further assessed. In the classical straw technique, no sperms were available following freezing-thawing of 1-10 sperms per straw. The cryopreservation of single sperm in the culture dish 
a

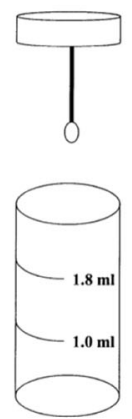

c

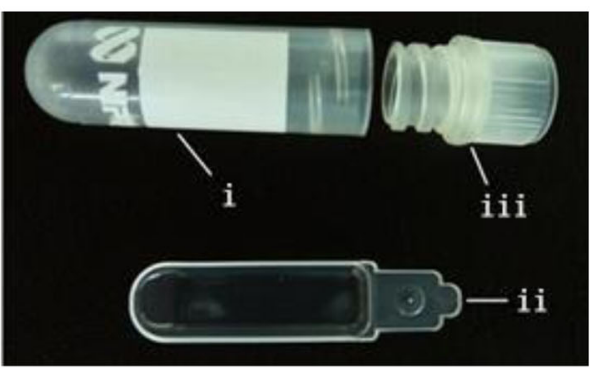

e

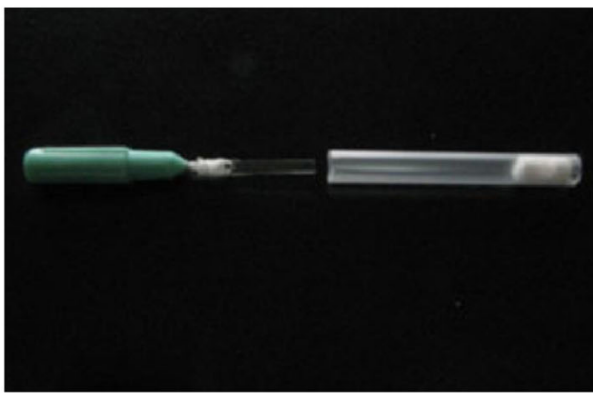

$\mathrm{g}$

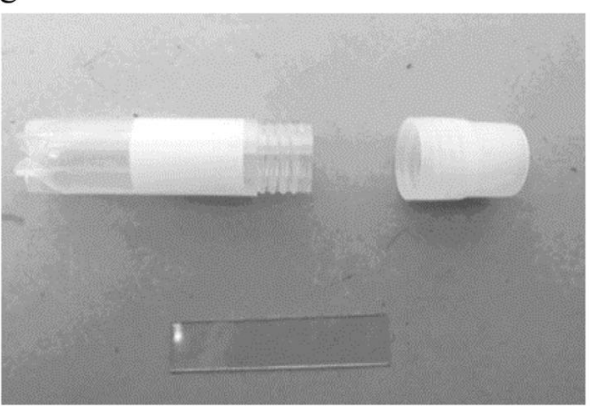

b

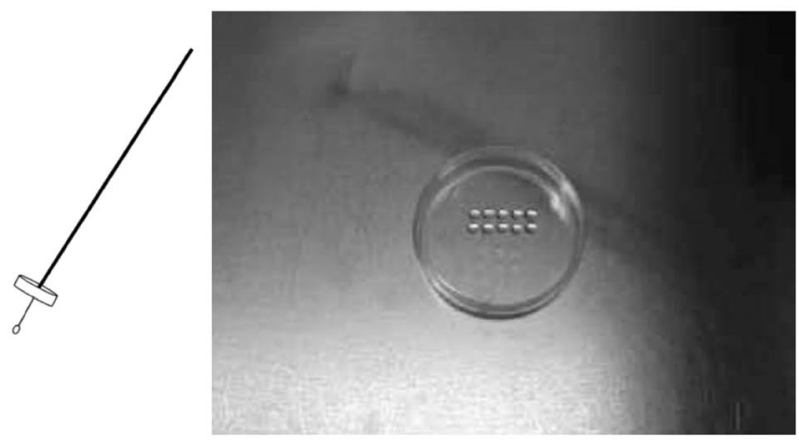

d

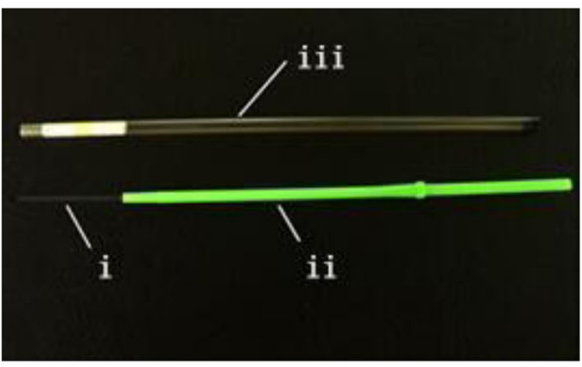

f

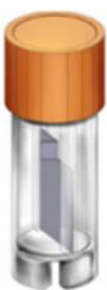

\section{Cryo-tube}
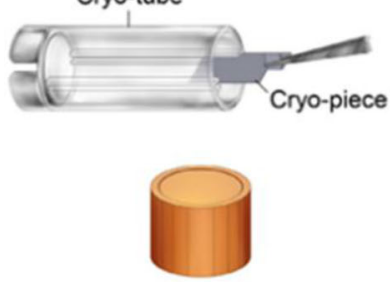

h

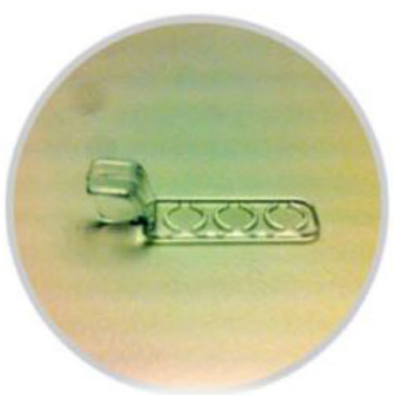

Fig. 3 (a) (Adapted from Schuster et al. [29]) Cryoloops can be magnetically attached to a metal wand for easier manipulation. (b) (Adapted from Sereni et al. [34]) Microdrop in a plastic dish. (c) (Adapted from Endo et al. [35]) Cell Sleeper vial (i) is equipped with an inner tray (ii) and a screw cap (iii). (d) (Adapted from Endo et al. [35]) Cryotop comprises a fine polypropylene strip (i), plastic handle (ii), and cover straw (iii). (e) (Adapted from Peng et al. [39]) A cryoleaf bound to a handle by cotton thread is placed to a protective casing containing cotton wool at the bottom. (f) (Adapted from Sun et al. [40]) Droplets and polypropylene piece of cryopiece system. (g) (Adapted from Ma et al. [41]) The closed slice frozen carrier comprised self-made non-toxic polypropylene flakes and conventional sperm cryovials. (h) (Adapted from Berkovitz et al. [43]) The sperm vitrification device 
achieved high recovery rates compared with the classical straw technique [33]. In 2008, Sereni et al. [34] used a similar technique to freeze individual testicular spermatozoa. The total number of frozen spermatozoa was 431 (2-300 spermatozoa/sample) across six patient samples. Cryoprotectant was added to the culture dish. Before freezing, the sperm motility rate was $3.5 \%$, and after thawing, $67 \%$ of motile sperm retained their motility. The sperm recovery rate was $100 \%$. A total of 51 oocytes were treated across the six study samples, and in two out of six cases, motile spermatozoa were injected. The fertilization rate was $18 \%$ [34].

This method is simple and easy to control, but polystyrene culture dishes with microdroplets are problematic because their size and shape make them more difficult to store for a long term in liquid nitrogen and they cannot be sealed to create a closed system. Therefore, the risk of potential cross-contamination may increase. To date, the clinical application of the cryopreservation of individual sperm in the culture dish remains limited.

\section{Cell sleeper}

There remains no consensus regarding the ideal carrier for the cryopreservation of individual or small quantities of spermatozoa for clinical purposes [15]. In 2012, Endo et al. $[35,36]$ cryopreserved small numbers of spermatozoa using the Cell Sleeper, which is a closed system. The Cell Sleeper is a vial-based cell-cryopreservation container equipped with an inner tray (Fig. 3c). Individual sperm were added to a droplet $(3.5 \mu \mathrm{L})$ on the tray. The tray was put into a vial and sealed with a screw cap. The vial was placed in $\mathrm{LN}_{2}$ vapor $\left(-120^{\circ} \mathrm{C}\right)$ before exposure to sterilized $\mathrm{LN}_{2}$. The authors applied this technique clinically in one NOA patient, resulting in the birth of a healthy boy. In addition, 12 spermatozoa from testis were vitrified. After thawing, 10 were successfully retrieved and injected individually into six mature oocytes, and the fertilization rate was $83 \%$ [35]. The effect of different vitrification volumes $(0.5,1.0$, and $3.5 \mu \mathrm{L})$ on individually vitrified spermatozoa was measured using the Cell Sleeper. The Cell Sleeper was placed $0.5 \mathrm{~cm}$ above the $\mathrm{LN}_{2}$ vapor for $2.5 \mathrm{~min}$ before exposure to sterilized $\mathrm{LN}_{2}$. After thawing, all spermatozoa were recovered and DNA integrity was maintained. The viable sperm rate was significantly higher when spermatozoa were frozen in a $3.5 \mu \mathrm{L}$ droplet rather than in $0.5 \mu \mathrm{L}(P<0.01)$. Frozen sperm were comparable with fresh sperm in terms of apoptotic DNA fragmentation [36]. In 2016, Coetzee et al. [37] used Cell Sleepers to cryopreserve the testicular sperm. Five to twenty-seven spermatozoa were added to a $2 \mu \mathrm{L}$ droplet of cryopreservation solution on the tray. The Cell Sleeper was placed $4-5 \mathrm{~cm}\left(-115^{\circ} \mathrm{C}--130^{\circ} \mathrm{C}\right)$ above the $\mathrm{LN}_{2}$ vapor for $2 \mathrm{~min}$ and then plunged into the $\mathrm{LN}_{2}$. A total of 304 spermatozoa were frozen in 20 Cell Sleepers, and of these,
265 were warmed. The sperm recovery rate was $94 \%$, and the motility rate was $56 \%$. Recovered thawed spermatozoa were injected into 179 mature oocytes, and the fertilization rate was 66\% [37]. Cryopreserved immotile testicular spermatozoa can be used to inject oocytes, thereby resulting in normal fertilization [60]. Therefore, the Cell Sleeper method is fast, easy, and simple to complete. However, one drawback is the time taken to search for the sperm after thawing, which takes approximately $30 \mathrm{~min}$ (this period is relatively longer than that in other procedures). In addition, microsurgery requires constant lifting and lowering of the ICSI needles, thereby leading to a higher risk of needle breakage.

\section{Cryotop}

Cryotop is a mature commercialized carrier. Cryotop, comprising fine polypropylene strips, a plastic handle, and a cover straw, has been intensely studied as a method to freeze single sperm in microdroplets (Fig. $3 \mathrm{~d}$ ). In previous studies, small numbers of spermatozoa were frozen using various types of containers. However, the lack of proper technology remains a drawback [15]. Cryotop acts as a vitrification container for both embryos and oocytes, and this system achieved 99\% post-thawing survival rates [61]. Cryotop methods are also suitable for the cryopreservation of small numbers of sperm. Endo et al. [35, 36, 38] developed a simple novel vitrification technique for a single spermatozoon with the use of Cryotop at room temperature. Individual sperm were transferred to a droplet of freezing medium $(1 \mu \mathrm{L})$ on the Cryotop strip, and the strip was immediately placed at approximately $4 \mathrm{~cm}$ above the surface of the $\mathrm{LN}_{2}$ for $2 \mathrm{~min}\left(-120^{\circ} \mathrm{C}\right)$, then directly exposed to sterilized $\mathrm{LN}_{2}$. Resuscitation was performed at $37^{\circ} \mathrm{C}$. One hundred spermatozoa frozen using Cryotop or empty ZP as containers had comparable rates of sperm recovery and motility after thawing. Two different cryoprotectants, SpermFreeze and sucrose, were tested, and similar rates of recovery after thawing were observed; however, the motility rate was significantly lower when sperm was frozen in SpermFreeze compared with sucrose. Sperm from ejaculates and testis were frozen using Cryotop, and the recovery and motility rates after thawing were $93 \%$ and $40 \%$, respectively [38]. The authors applied this technique clinically in two patients with severe oligozoospermia or nonobstructive azoospermia (NOA). Eighty-one spermatozoa from testis in 8 containers (10.1 sperm per container) were vitrified, and 10 of these were warmed. All were successfully retrieved and injected individually into four mature oocytes. The fertilization rate was 75\% [35]. This shows that the Cryotop method can effectively cryopreserve testicular sperm collected by micro-TESE. In another study, spermatozoa from ejaculates were cryopreserved in the Cryotop and the Cell Sleeper. After thawing, sperm recovery rate was 
relatively high ( $\geq 96 \%)$ in both container groups. Motility and viability of spermatozoa were similar between the two containers [36]. In 2015, Chen et al. [62] vitrified ten human spermatozoa without CPAs in $0.5 \mu \mathrm{L}$ of freezing medium on a Cryotop strip. Both sperm recovery and motility between the sperm cryopreserved without CPAs and/or sucrose showed no significant differences. Thawed sperms without CPAs displayed higher viability and lower chromatin or DNA damage compared with samples cryopreserved with sucrose.

Cryotop is a non-biological material and does not pose any ethical issues. This technique is particularly valuable to patients with severe azoospermia and oligoazoospermia. Cryotops are now employed in $\geq 40$ countries, and their use is documented in $\geq 100,000$ clinical cases of oocyte and embryo cryopreservation (personal communication from Kitazato Biophrma) [38]. Healthy babies have been born with the aid of the Cryotop method.

Currently, the Cryotop vector is mainly used for freezing embryos and for freezing rare spermatozoa. In recent years, to the best of our knowledge, some new carriers, including cryoleaf, cryopiece, and closed slice, have been designed on the basis of the Cryotop method in China.

\section{Cryoleaf}

In 2011, one study [39] demonstrated a novel method for freezing and recovery of individual or small numbers of spermatozoa. Small numbers of sperms were cryopreserved on a self-designed cryoleaf under high humidity conditions cryoprotectant that prevents evaporation (Fig. 3e). Individual spermatozoa were transferred into a droplet $(0.2 \mu \mathrm{L})$ comprising $0.1 \mu \mathrm{L}$ seminal plasma and $0.1 \mu \mathrm{L}$ cryoprotectant. The cryoleaf was subsequently sheathed with a casing and then slowly lowered $(20 \mathrm{~cm}$ in $30 \mathrm{~s})$ to $1 \mathrm{~cm}$ over LN2 surface, exposed to the vapor for $2 \mathrm{~min}$, and then plunged into the $\mathrm{LN}_{2}$. In this method, sperm obtained through percutaneous epididymal punctures had a motility recovery of 93\% after freezing and rewarming; ejaculated sperm had a motility recovery of $62 \%$. Following ICSI injection, no differences in the rates of fertilization were observed between fresh and frozen-thawed spermatozoa and the cleavage rates were almost similar [39]. Therefore, the cryoleaf method can effectively and conveniently cryopreserve individual sperms. However, skilled operators are required, and the freezing process must be rapid to prevent evaporation of the droplets. The technical requirements of this technique limit its widespread use in clinical practice. No successful pregnancy has been reported using this technique.

The cryoleaf vector have limited clinical use owing to their shortcomings, and the cryoleaf vector was consequently replaced by the cryopiece vector in 2016 .

\section{Cryopiece}

In previous studies [37, 38], the Cryotop and cryoleaf were developed to store the spermatozoa obtained from patients with severe oligozoospermia. Considering the potential risk of cross-contamination in $\mathrm{LN}_{2}$ and possible fertilization failure, a novel high-efficiency storage device and system, cryopiece technology, were developed to cryopreserve individual sperm or small sperm numbers. The cryopiece carrier (Fig. 3f) has been commercialized in China. Using this method, Sun et al. [40] from the Shanghai First People's Hospital reported the birth of the first single-sperm cryotube baby in China in 2016. They applied this technique clinically in four patients with severe oligozoospermia or NOA. Spermatozoa with normal morphologies and higher motility were added to droplets on the cryopiece. The freezing tubes were placed into $\mathrm{LN}_{2}$ vapor for $15 \mathrm{~min}$ and subsequently plunged in $\mathrm{LN}_{2}$. After thawing, $83 \%$ of the spermatozoa completely recovered with movement rate of $48 \%$. Following ICSI, fertilization rate was 73\%, and 19 (86\%) zygote cleavage events were observed. Four healthy babies were born at term [40].

\section{Closed slice}

The microdrop single-sperm freezing method has been effectively employed in clinical practice. In 2015, Ma et al. [41] employed the closed slice method to rapidly freeze individual sperms in 2015. The frozen carrier comprised self-made non-toxic polypropylene flakes and conventional sperm cryovials (Fig. 3g). The effects of different vitrification volumes $(0.5,1.0$, and $3.5 \mu \mathrm{L})$ on individually vitrified spermatozoa were compared using the closed slice method. Ten forward-moving spermatozoa were placed into droplets at $1 \mathrm{~cm}$ from the surface of the $\mathrm{LN}_{2}$ for $2 \mathrm{~min}$, followed by $\mathrm{LN}_{2}$ plunging. After thawing, no significant differences were observed in the sperm recovery rate, motility rate, or viability rate among the three groups. Compared with techniques using frozen protective agents and those lacking cryoprotectants, the sperm recovery rate, activity rate, and survival rate were not statistically different. Closed slice vitrification methods could effectively freeze the testicular sperm in patients with obstructive azoospermia, and these methods showed a high sperm recovery and survival rate after thawing $(92 \%$ and $66 \%$ ). The frozen and fresh groups had comparable cleavage and fertilization rates [42].

\section{The novel sperm vitrification device (SpermVD)}

The novel SpermVD represents an efficient carrier to freeze small numbers of spermatozoa, which optimizes fertility preservation (Fig. 3h). The SpermVD is similar to cryopiece. Sperm can be transferred to $0.8-1 \mu \mathrm{L}$ microdroplets on the SpermVD, and $3.6 \mathrm{~mL}$ cryovials containing the SpermVD can be submerged into $\mathrm{LN}_{2}$. 
The time to search for spermatozoa after thawing was shortened from hours to minutes, and the sperm recovery rate reached $96 \%$. The motility rate was $33 \%$. The sperm were successfully used for fertilization. The fertilization and pregnancy rates were reported to be $59 \%$ and $55 \%$, respectively. The delivery rate was $32 \%$, and the miscarriage rate was $29 \%$ [43]. The cryopreservation of motile sperm using the novel SpermVD vector yielded improved clinical outcomes but immotile sperm were not preserved using this device, thereby limiting the knowledge regarding the efficacy of the SpermVD for immotile sperm from this source.

\section{Conclusions}

In the last few decades, several studies have suggested a declining trend of human semen quality [63-67]. Some genetic and pathological factors are known [6], but the exact cause remains unclear. Environmental and/or occupational factors along with lifestyle practices could contribute to the deterioration of semen quality [68-71]. Lifestyle factors include cigarette smoking, alcohol intake, diet, obesity, use of illicit drugs, advanced paternal age, psychological stress, and caffeine intake. Individuals are exposed to each risk factor for varying durations and degrees of severity [72]. Awareness and recognition of the possible impact of risk factors present in daily life is crucial. In special situations, cryopreservation of sperm is also a way to avoid risks. Perfecting freezing technology will ensure better freezing effects with different qualities of semen. For example, patients with severe oligozoospermia and nonobstructive azoospermia have very limited numbers of viable sperm. Conventional cryopreservation techniques are inadequate for preserving individually selected sperm, but single-sperm freezing technology gives hope to these patients.

Since the inception of single-sperm freezing, studies on carriers and freezing methods have improved the recovery and activity rates of sperms after cryopreservation. From the use of the initial empty ZP biocarriers to the current non-biological carriers, different types of carriers have been designed for freezing small numbers of spermatozoa under different laboratory conditions. Although sperm using a single sperm freezing method by assisted reproductive technology can result in a healthy baby, to date, an ideal container that can be universally used is yet to be developed. Each cryopreservation method has its own advantages and disadvantages. As a non-biological carrier, the empty capsule freezes single sperm, promotes high sperm recovery, and high sperm movement rates. Moreover, the time to search for the recovered sperm is short. However, owing to the complexity of vector production and the lack of methods to effectively preserve the carrier, the development of the non-biological carriers is slow. Further, single-sperm microdroplet freezing methods were developed, with frozen carriers composed of polystyrene and polypropylene representing safe and simple manufacturing procedures that reduce experimental costs. In these methods, the micromanipulation process is convenient and sperm recovery rates, movement rates, and fertilization rates are high. However, this technique has not been widely used because skilled operators are required to prevent the evaporation of the droplets in clinical settings, the recovery process and the time to search for sperm are prolonged, and there is paucity of clinical data on its effectiveness. Current evidence fails to completely support the benefit of one technology over another. Every frozen sperm sample is at a risk of being contaminated. This contamination could be caused by the sample itself carrying viruses and bacteria, the non-sealed nature of the frozen carrier, and non-clean liquid nitrogen. Therefore, patients who store sperm need to be screened for the presence of HIV, hepatitis B and C, syphilis, and bacterial infections. Furthermore, the materials of the infected cases should not be stored or they should be stored in separate storage tanks, reducing the risk of virus transmission. However, the risk of contamination cannot be completely eliminated because of the possibility that patients cannot be screened for a disease. If the patient is infected, each straw may contain many viral particles. Washing the spermatozoa by a buoyant density gradient and resuspending them in a suitable sterile medium can reduce the presence of the virus below detectable levels [73]. From a safety point of view, a sealed refrigerated carrier should be used to ensure that external contamination is avoided. The laboratory must have standard operating procedures for cleaning and maintaining liquid nitrogen containers to minimize the possibility of environmental pollution [74]. Compared with ordinary sperm vitrification, single-sperm cryopreservation method uses ICSI needles to add sperm to a sterile cryoprotectant, which reduces the potential risk of virus contamination. However, due to the low rate of clinical use, there is no data on the sperm transmission contamination of the single-sperm cryopreservation method.

\section{Future perspectives}

Because individual sperm freezing is beneficial for patients with poor sperm quality and azoospermia, early sperm optimization is of particular importance. To date, the majority of methods use traditional density gradient centrifugation to optimize sperm yields. This highlights the large developmental potential of these procedures and the need to improve the methods of single-sperm freezing technology in the future.

An array of cryoprotectants has been described. The major component of these protectants is glycerin, which reduces freezing damage and improves sperm quality. 
To date, however, there have been limited improvements in the use of cryoprotectants. During single-sperm droplet freezing, no cryoprotectant has achieved optimal freezing results, mainly owing to the freezing procedure and droplet volume. In-depth studies are required to establish clear reference standards.

In future research, well-designed clinical trials are required for assessing the feasibility and efficiency of various spermatozoa freezing methodologies. The goal is to provide health clinics with high-quality sperm for assisted reproduction. Further developments in this area can reduce the fertility treatment cycle, thereby improving both fertilization and pregnancy rates.

\section{Abbreviations}

CPA: Cryoprotective agent; HA: Hyaluronan; ICSI: Intracytoplasmic sperm injection; LN $\mathrm{N}_{2}$ : Liquid nitrogen; NOA: Nonobstructive azoospermia; TESE: Testicular sperm extraction; VD: Vitrification device; ZP: Zona pellucida

\section{Acknowledgements}

Not applicable.

\section{Authors' contributions}

SL and FL conceived the idea. SL was involved in literature collection. Two authors independently and critically reviewed the selected manuscripts. SL contributed to the first draft, which was then expanded and improved by two authors. The two authors reviewed and approved the completed manuscript.

\section{Funding}

No funding was requested for this review.

\section{Availability of data and materials}

Literature research results are available from the authors upon reasonable request.

\section{Ethics approval and consent to participate}

Not applicable.

\section{Consent for publication}

Not applicable.

\section{Competing interests}

The authors declare that they have no competing interests.

Received: 3 March 2020 Accepted: 29 April 2020

Published online: 12 May 2020

\section{References}

1. Checa Vizcaíno MA, González-Comadran M, Jacquemin B. Outdoor air pollution and human infertility: a systematic review. Fertil Steril. 2016;106: 897-904.

2. Bártolo A, Reis S, Monteiro S, Leite R, Montenegro N. Psychological adjustment of infertile men undergoing fertility treatments: an association with sperm parameters. Arch Psychiat Nurs. 2016:30:521-6.

3. Jackson RE, Bormann CL, Hassun PA, Rocha AM, Motta EL, Serafini PC, et al. Effects of semen storage and separation techniques on sperm DNA fragmentation. Fertil Steril. 2010;94:2626-30.

4. Tschudin S, Bitzer J. Psychological aspects of fertility preservation in men and women affected by cancer and other life-threatening diseases. Hum Reprod Update. 2009;15:587-97.

5. Kenney LB, Antal Z, Ginsberg JP, Hoppe BS, Bober SL, Yu RN, et al. Improving male reproductive health after childhood, adolescent, and young adult Cancer: Progress and future directions for survivorship research. J Clin Oncol. 2018;36:2160-8.

6. Krausz C. Male infertility: pathogenesis and clinical diagnosis. Best Pract Res Clin Endocrinol Metab. 2011;25:271-85.
7. Palermo G, Joris H, Devroey P, Van Steirteghem AC. Pregnancies after intracytoplasmic injection of single spermatozoon into an oocyte. Lancet. 1992;340:17-8.

8. Sherman JK. Synopsis of the use of frozen human semen since 1964:state of the art of human semen banking. Fertil Steril. 1973;24:397-412.

9. Hewitson L, Martinovich C, Simerly C, Takahashi D, Schatten G. Rhesus offspring produced by intracytoplasmic injection of testicular sperm and elongated spermatids. Fertil Steril. 2002;77:794-801.

10. Park YS, Lee SH, Song SJ, Jun JH, Koong MK, Seo JT. Influence of motility on the outcome of in vitro fertilization/intracytoplasmic sperm injection with fresh vs. frozen testicular sperm from men with obstructive azoospermia. Fertil Steril. 2003;80:526-30.

11. Miller N, Biron-Shental T, Pasternak Y, Belenky MM, Shefi S, Itsykson P, et al. Fertility outcomes after extended searches for ejaculated spermatozoa in men with virtual azoospermia. Fertil Steril. 2017:107:1305-11.

12. Schlegel PN, Su LM. Physiological consequences of testicular sperm extraction. Hum Reprod. 1997:12:1688-92.

13. Andersson AM, Jørgensen $N$, Frydelund-Larsen $L$, Rajpert-De Meyts $E$, Skakkebaek NE. Impaired Leydig cell function in infertile men: a study of 357 idiopathic infertile men and 318 proven fertile controls. J Clin Endocrinol Metab. 2004:89:3161-7.

14. Nawroth F, Isachenko V, Dessole S, Rahimi G, Farina M, Vargiu N, et al. Vitrification of human spermatozoa without cryoprotectants. Cryo Lett. 2002;23:93-102

15. AbdelHafez FA, Bedaiwy M, El-Nashar SA, Sabanegh E, Desai N. Techniques for cryopreservation of individual or small numbers of human spermatozoa: a systematic review. Hum Reprod Update. 2009;15:153-64.

16. Cohen J, Garrisi GJ, Congedo-Ferrara TA, Kieck KA, Schimmel TW, Scott RT. Cryopreservation of single human spermatozoa. Hum Reprod. 1997;12:9941001.

17. Walmsley R, Cohen J, Ferrara-Congedo T, Reing A, Garrisi J. The first births and ongoing pregnancies associated with sperm cryopreservation within evacuated egg zonae. Hum Reprod. 1998;13(Suppl. 4):61-70.

18. Montag M, Rink K, Dieckmann U, Delacrétaz G, van der Ven H. Laser-assisted cryopreservation of single human spermatozoa in cell-free zona pellucida. Andrologia. 1999:31:49-53.

19. Borini A, Sereni E, Bonu C, Flamigni C. Freezing a few testicular spermatozoa retrieved by TESA. Mol Cell Endocrinol. 2000;169:27-32.

20. Hsieh Y, Tsai H, Chang C, Lo H. Cryopreservation of human spermatozoa within human or mouse empty zona pellucidae. Fertil Steril. 2000;73:694-8.

21. Liu J, Zheng XZ, Baramki TA, Compton G, Yazigi RA, Katz E. Cryopreservation of a small number of fresh human testicular spermatozoa and testicular spermatozoa cultured in vitro for 3 days in an empty zona pellucida. J Androl. 2000;21:409-13.

22. Fusi F, Calzi F, Rabellotti E, Papaleo E, Gonfiantini C, Bonzi V, et al. Fertilizing capability of frozen-thawed spermatozoa recovered from microsurgical epididymal sperm aspiration and cryopreserved in oocyte-free human zona pellucida. Hum Reprod. 2001;16:117.

23. Levi-Setti PE, Albani E, Negri L, Cesana A, Novara P, Bianchi S. Cryopreservation of a small number of spermatozoa in yolk-filled human zonae pellucidae. Arch Ital Urol Androl. 2003;75:195-8.

24. Just A, Gruber I, Wöber M, Lahodny J, Obruca A, Strohmer H. Novel method for the cryopreservation of testicular sperm and ejaculated spermatozoa from patients with severe oligospermia: a pilot study. Fertil Steril. 2004;82:445-7.

25. Herrler A, Eisner S, Bach V, Weissenborn U, Beier HM. Cryopreservation of spermatozoa in alginic acid capsules. Fertil Steril. 2006;85:208-13.

26. Isaev DA, Zaletov SY, Zaeva W, Zakharova EE, Shafei RA, Krivokharchenko IS. Artificial microcontainers for cryopreservation of solitary spermatozoa. Hum Reprod. 2007;22:1154.

27. Araki Y, Yao T, Asayama Y, Matsuhisa A, Araki Y. Single human sperm cryopreservation method using hollow-core agarose capsules. Fertil Steril. 2015;104:1004-9.

28. Tomita K, Sakai S, Khanmohammadi M, Yamochi T, Hashimoto S, Anzai M, et al. Cryopreservation of a small number of human sperm using enzymatically fabricated, hollow hyaluronan microcapsules handled by conventional ICSI procedures. J Assist Reprod Genet. 2016:33:501-11.

29. Schuster TG, Keller LM, Dunn RL, Ohl DA, Smith GD. Ultra-rapid freezing of very low numbers of sperm using cryoloops. Hum Reprod. 2003;18:788-95.

30. Desai NN, Blackmon H, Goldfarb J. Single sperm cryopreservation on cryoloops: an alternative to hamster zona for freezing individual spermatozoa. Reprod BioMed Online. 2004;9:47-53. 
31. Desai N, Culler C, Goldfarb J. Cryopreservation of single sperm from epididymal and testicular samples on cryoloops: preliminary case report. Fertil Steril. 2004;82:S264-5.

32. Quintans CJ, Donaldson MJ, Asprea I, Geller M, Rocha M, Pasqualini RS. Development of a novel approach for cryopreservation of very small numbers of spermatozoa. Hum Reprod. 2000;15:99.

33. Bouamama N, Briot P, Testart J. Comparison of two methods of cryoconservation of sperm when in very small numbers. Gynecol Obstet Fertil. 2003;31:132-5.

34. Sereni E, Bonu MA, Fava L, Sciajno R, Serrao L, Preti S, et al. Freezing spermatozoa obtained by testicular fine needle aspiration: a new technique. Reprod BioMed Online. 2008;16:89-95.

35. Endo Y, Fujii Y, Kurotsuchi S, Motoyama H, Funahashi H. Successful delivery derived from vitrified-warmed spermatozoa from a patient with nonobstructive azoospermia. Fertil Steril. 2012;98:1423-7.

36. Endo Y, Fujii Y, Shintani K, Seo M, Motoyama H, Funahashi H. Simple vitrification for small numbers of human spermatozoa. Reprod BioMed Online. 2012;24:301-7.

37. Coetzee K, Ozgur K, Berkkanoglu M, Bulut H, Isikli A. Reliable single sperm cryopreservation in cell sleepers for azoospermia management. Andrologia. 2016:48:203-10

38. Endo Y, Fujii Y, Shintani K, Seo M, Motoyama H, Funahashi H. Single spermatozoon freezing using cryotop. J Mamm Ova Res. 2011;28:47-52.

39. Peng QP, Cao SF, Lyu QF, Xue SG, Jin W, Liu XY, et al. A novel method for cryopreservation of individual human spermatozoa. In Vitro Cell Dev Biol Anim. 2011;47:565-72.

40. Sun J, Chen W, Zhou L, Hu J, Li Z, Zhang Z, et al. Successful delivery derived from cryopreserved rare human spermatozoa with novel cryopiece. Andrology. 2017:5:832-7

41. Ma C, Hu MG, Wang ZT. Study on ultra-rapid vitrification of human microsperm by closed-sheet method. Mod Obstet Gynecol Prog. 2015;24:762-4.

42. Ma C, Hu MG, Jin B. Application of closed slice method in cryopreservation of testicular spermatozoa. Contemp Chin Med. 2017;24:19-21.

43. Berkovitz A, Miller N, Silberman M, Belenky M, Itsykson P. A novel solution for freezing small numbers of spermatozoa using a sperm vitrification device. Hum Reprod. 2018;33:1975-83.

44. Patrizio P, Ord T, Balmaceda JP, Asch RH. Successful fertilization, pregnancy, and birth using epididymal sperm frozen 24 hours after conventional oocyte insemination. Fertil Steril. 1995;64:863-5.

45. Podsiadly BT, Woolcott RJ, Stanger JD, Stevenson K. Pregnancy resulting from intracytoplasmic injection of cryopreserved spermatozoa recovered from testicular biopsy. Hum Reprod. 1996;11:1306-8.

46. Hewitt J, Cohen J, Mathew T, Rowland G. Cryopreservation of semen in patients with malignant disease: role of in-vitro fertilisation. Lancet. 1985;2:446-7.

47. Hsieh YY, Tsai HD, Chang CC, Lo HY. Sperm cryopreservation with empty human or mouse zona pellucidae. A comparison J Reprod Med. 2000;45:383-6.

48. Zhu W, Huang M, Xing F. A small amount of human sperm is cryopreserved as a frozen storage carrier with empty zona pellucida. Reprod Contracept. 2002;22:338-41.

49. Camaioni A, Russo MA, Odorisio T, Gandolfi F, Fazio VM, Siracusa G. Uptake of exogenous DNA by mammalian spermatozoa: specific localization of DNA on sperm heads. J Reprod Fertil. 1992;96:203-12.

50. Spadafora C. Sperm cells and foreign DNA: a controversial relation. BioEssays. 1998;20:955-64.

51. Guyomard C, Rialland L, Fremond B, Chesne C, Guillouzo A. Influence of alginate gel entrapment and cryopreservation on survival and xenobiotic metabolism capacity of rat hepatocytes. Toxicol Appl Pharmacol. 1996;141:349-56.

52. Stensvaag $V$, Furmanek T, Lønning $K$, Terzis AJ, Bjerkvig R, Visted T. Cryopreservation of alginate-encapsulated recombinant cells for antiangiogenic therapy. Cell Transplant. 2004;13:35-44.

53. Sakai S, Kawabata K, Tanaka S, Harimoto N, Hashimoto I, Mu C, et al. Subsievesize agarose capsules enclosing ifosfamide-activating cells: a strategy toward chemotherapeutic targeting to tumors. Mol Cancer Ther. 2005;4:1786-90.

54. Tuvikene R, Truus K, Kollist A, Volobujeva O, Mellikov E, Pehk T. Gel-forming structures and stages of red algal galactans of different sulfation levels. J Appl Phycol. 2008;20:527-35.

55. Isachenko E, Isachenko V, Katkov II, Rahimi G, Schöndorf T, Mallmann P, et al. DNA integrity and motility of human spermatozoa after standard slow freezing versus cryoprotectant-free vitrification. Hum Reprod. 2004;19:932-9.

56. Lane M, Schoolcraft WB. Gardner DK. Vitrification of mouse and human blastocysts using a novel cryoloop container-less technique Fertil Steril. 1999;72:1073-8.
57. Mukaida T, Nakamura S, Tomiyama T, Wada S, Kasai M, Takahashi K. Successful birth after transfer of vitrified human blastocysts with use of a cryoloop containerless technique. Fertil Steril. 2001;76:618-20.

58. Liebermann J, Tucker MJ, Sills ES. Cryoloop vitrification in assisted reproduction: analysis of survival rates in $>1000$ human oocytes after ultrarapid cooling with polymer augmented cryoprotectants. Clin Exp Obstet Gynecol. 2003;30:125-9.

59. Mukaida T, Takahashi K, Kasai M. Blastocyst cryopreservation: ultrarapid vitrification using cryoloop technique. Reprod BioMed Online. 2003;6:221-5.

60. Ohlander S, Hotaling J, Kirshenbaum E, Niederberger C, Eisenberg ML. Impact of fresh versus cryopreserved testicular sperm upon intracytoplasmic sperm injection pregnancy outcomes in men with azoospermia due to spermatogenic dysfunction: a meta-analysis. Fertil Steril. 2014;101:344-9.

61. Kuwayama M, Vajta G, Kato O, Leibo SP. Highly efficient vitrification method for cryopreservation of human oocytes. Reprod BioMed Online. 2005;11: 300-8.

62. Chen Y, Li L, Qian Y, Xu C, Zhu Y, Huang H, et al. Small-volume vitrification for human spermatozoa in the absence of cryoprotectants by using Cryotop. Andrologia. 2015;47:694-9.

63. Carlsen E, Giwercman A, Keiding N, Skakkebaek NE. Evidence for decreasing quality of semen during past 50 years. BMJ. 1992;305:609-13.

64. Jørgensen N, Andersen AG, Eustache F, Irvine DS, Suominen J, Petersen JH, et al. Regional differences in semen quality in Europe. Hum Reprod. 2001;16: 1012-9.

65. Aitken JR. Falling sperm counts twenty years on: where are we now? Asian Androl. 2013:15:204-7.

66. Levine $H$, Jørgensen N, Martino-Andrade A, Mendiola J, Weksler-Derri D, Mindlis I, et al. Temporal trends in sperm count: a systematic review and meta-regression analysis. Hum Reprod Update. 2017;23:646-59.

67. Sengupta P, Borges E, Dutta S, Krajewska-Kulak E. Decline in sperm count in European men during the past 50 years. Hum Exp Toxicol. 2018;37:247-55.

68. Jurewicz J, Radwan M, Sobala W, Radwan P, Bochenek M, Hanke W. Effects of occupational exposure - is there a link between exposure based on an occupational questionnaire and semen quality? Syst Biol Reprod Med. 2014; 60:227-33.

69. Knez J. Endocrine-disrupting chemicals and male reproductive health. Reprod BioMed Online. 2013;26:440-8.

70. Bonde JP. Occupational causes of male infertility. Curr Opin Endocrinol Diabetes Obes. 2013;20:234-9.

71. Sharma R, Biedenharn KR, Fedor JM, Agarwal A. Lifestyle factors and reproductive health: taking control of your fertility. Reprod Biol Endocrinol. 2013;11:66

72. Durairajanayagam D. Lifestyle causes of male infertility. Arab J Urol. 2018;16: 10-20.

73. McKee TA, Avery S, Majid A, Brinsden PR. Risks for transmission of hepatitis C virus during artificial insemination. Fertil Steril. 1996;66:161-3.

74. Avery SM, McLaughlin EA, Dawson KJ. Safe cryopreservation of sperm and embryos. Hum Fertil. 1998;1:84-6.

\section{Publisher's Note}

Springer Nature remains neutral with regard to jurisdictional claims in published maps and institutional affiliations.

Ready to submit your research? Choose BMC and benefit from:

- fast, convenient online submission

- thorough peer review by experienced researchers in your field

- rapid publication on acceptance

- support for research data, including large and complex data types

- gold Open Access which fosters wider collaboration and increased citations

- maximum visibility for your research: over $100 \mathrm{M}$ website views per year

At $\mathrm{BMC}$, research is always in progress.

Learn more biomedcentral.com/submission 